\title{
A hemagglutinin quantification method for development of an influenza pandemic vaccine using size exclusion high performance liquid chromatography
}

\author{
HANG SIK ROH ${ }^{1}$, HYE MIN SONG $^{1}$, BO REUM YUN ${ }^{1}$, HYUN KYUNG KANG $^{1}$, KEUM SUK CHOI $^{1}$, \\ YUN JU PARK ${ }^{1}$, DONG SUB KIM ${ }^{1}$, SEUNG HEE KIM ${ }^{1}$, IN PIL MO ${ }^{2}$, BEUM-SOO AN ${ }^{3}$ and CHI YOUNG AHN ${ }^{1}$ \\ ${ }^{1}$ Biologics Research Division, National Institute of Food and Drug Safety Evaluation, Korea Food and Drug Administration, \\ Cheongwon-gun, Chungcheongbuk-do 363-951; ${ }^{2}$ College of Veterinary Medicine, Chungbuk National University, Cheongju-si, \\ Chungcheongbuk-do 361-763; ${ }^{3}$ Department of Biomaterials Science, College of Natural Resources and Life Science, \\ Pusan National University, Miryang, Gyeongsangnam-do 627-706, Republic of Korea
}

Received February 17, 2014; Accepted November 19, 2014

DOI: $10.3892 / \mathrm{mmr} .2014 .3049$

\begin{abstract}
Single radial immunodiffusion (SRID) assay requires a reference antigen and an antibody to the hemagglutinin (HA) of an influenza vaccine. As it takes 2-3 months to develop the reference antigen, vaccine development is delayed in cases of an influenza pandemic. In the present study, the measurement of the HA content of influenza vaccines was assessed using size exclusion high performance liquid chromatography (SE-HPLC) for the rapid development of a pandemic vaccine. When the $2009 \mathrm{H} 1 \mathrm{~N} 1$ reference antigen, pandemic $2009 \mathrm{H} 1 \mathrm{~N} 1$ vaccine and 2010 seasonal influenza vaccines were analyzed by SE-HPLC, the HA of the reference antigen and vaccines was specifically separated. The presence and specificity of HA were evidenced with immunoprecipitation and ELISA assays. For the influenza vaccines, the chromatogram pattern and retention time of HA were similar among the antigen types (2009 H1N1, 2010 H3N2 and 2010 B). In addition, when SE-HPLC was applied, the ratio of HA chromatogram to peak area revealed a significant correlation with $\mathrm{HA}$ concentration for the reference antigen and vaccine. The result of the HA content
\end{abstract}

Correspondence to: Dr Chi Young Ahn, Biologics Research Division, National Institute of Food and Drug Safety Evaluation, Korea Food and Drug Administration, 187 Osongsaengmyeong 2-ro, Cheongwon-gun, Chungcheongbuk-do 363-951, Republic of Korea E-mail: 72notul@korea.kr

Professor Beum-soo An, Department of Biomaterials Science, College of Natural Resources and Life Science, Pusan National University, 50 Cheonghak-ri, Samnangjin-eup, Miryang, Gyeongsangnam-do 627-706, Republic of Korea

E-mail: anbs@pusan.ac.kr

Key words: pandemic, influenza vaccine, hemagglutinin, size exclusion high performance liquid chromatography, single radial immunodiffusion calculation based on SE-HPLC exhibited 99.91-100\% similarity, compared with that of SRID. These findings suggest that the measurement of peak area ratio/HA content using SE-HPLC may be a substitute for SRID and rapidly measure HA content to enable faster development of a vaccine during an influenza pandemic.

\section{Introduction}

Influenza is a respiratory disease, which is caused by an infectious virus. It can be effectively prevented by antibodies formed as a result of vaccination. An influenza vaccine can be developed using hemagglutinin (HA), present on the viral surface, which is a main antigen of the influenza virus. The HA content of an influenza virus is currently measured using a single radial immunodiffusion (SRID) assay, which is internationally authorized by the European Medicines Agency, Food and Drug Administration and World Health Organisation (1-5). However, this assay has the disadvantages of requiring the corresponding reference antigen and antiserum for the vaccine, and these reference factors require a longer time to develop (6-11). Due to these factors, vaccine development may be delayed, as revealed in the 2009 influenza pandemic. To overcome this issue, various methods have been investigated to develop a more rapid measurement of the HA content of an influenza vaccine. For example, Kapteyn et al $(7,8)$ measured HA content using reverse phase-high performance liquid chromatography. In addition, during the 2009 influenza pandemic, Li et al (12), measured HA content using SDS-PAGE and densitometry, which resulted in an $88-122 \%$ similarity with that of the conventional SRID for four subtypes of influenza vaccine (H1N1, H3N2, H5N1 and B type). Based on this study, the authors manufactured the first vaccine for the 2009 influenza pandemic (12). In the present study, size exclusion high performance liquid chromatography (SE-HPLC) was examined to develop a novel measurement method for HA content, which can be used without the preparation of reference antigen and antiserum. 


\section{Materials and methods}

Influenza vaccine and reference virus samples. The 2009 pandemic A/California/7/2009 (H1N1)v NYMC-X179A (2009 H1N1; Green Cross Corp., Yongin, Korea) vaccine, the A/California/7/2009 (H1N1) NYMC-X181 (2010 H1N1, Green Cross Corp.) monovalent seasonal vaccine, the A/Victoria/210/2009 (H3N2) NYMC-X187 (2010 H3N2, Green Cross Corp.) monovalent seasonal vaccine, the B/Brisbane/60/2008 (2010 B, Green Cross Corp.) monovalent seasonal vaccines and the 2010 trivalent vaccine (combined 2010 H1N1, 2010 H3N2 and 2010 B monovalent vaccines, Green Cross Corp.) were assessed in the present study. Reference antigens for 2009 H1N1 [National Institute for Biological References and Control (NIBSC) code: 09/146], 2010 H1N1 (NIBSC code: 09/294), 2010 H3N2 (NIBSC code: 10/102), 2010 B (NIBSC code: 8/352) were provided by NIBSC (Potters Bar, UK). The assessed vaccines, reference antigens and antiserums are listed in Table I.

SRID. The SRID assay was conducted using reference antigens and antiserums (Table I) provided by the NIBSC, which were appropriate for the influenza vaccines used in the present study, following the standard operating procedure of the National Institute of Food and Drug Safety evaluation and the Korea Food and Drug administration (Cheongwon-gun, Korea) as previously reported by Wood and Levandowski (13).

SE-HPLC. HPLC Alliance 2695 (Waters, Co., Milford, MA, USA), Waters 2489 UV-Vis Detector (Waters, Co.) and TSK

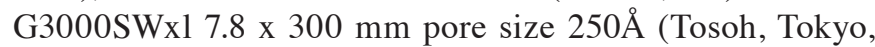
Japan) were used for HPLC. For the mobile phase, phosphate buffer (Sigma-Aldrich, St. Louis, MO, USA) was used at a flow rate of $1.0 \mathrm{ml} / \mathrm{min}$. The experimental condition was as follows: Sample injection volume, $20 \mu \mathrm{l}$; wavelength, $210 \mathrm{~nm}$ and temperature, $15^{\circ} \mathrm{C}$.

HA chromatographic peak. Subsequent to the vaccines and the corresponding reference antigens being assessed using HPLC, elutes of each peak in the chromatogram were collected and then the accuracy of HA level in each peak was examined using ELISA and immunoprecipitation.

ELISA. Fractions of the peak from the HPLC results were plated on a Nunc-Immuno MaxiSorp ${ }^{\mathrm{TM}}$ plate (Sigma-Aldrich) and incubated for $2 \mathrm{~h}$ at $37^{\circ} \mathrm{C}$. Following incubation of the fractions, the supernatants were removed and the plates were treated with anti-A/California/07/2009 antiserum (anti-H1N1 serum, NIBSC code: 09/152) (1:20,000 in phosphate-buffered saline (PBS)-Tween) for $2 \mathrm{~h}$ at $37^{\circ} \mathrm{C}$. The plates were then washed with PBS-Tween three times and secondary antibody (rabbit polyclonal secondary antibody to sheep immunoglobulin G-horseradish peroxidase; Abcam, Cambridge, UK) was applied for $2 \mathrm{~h}$ at $37^{\circ} \mathrm{C}$. SigmaFast ${ }^{\mathrm{TM}} o$-phenylenediamine (Sigma-Aldrich) was added and the intensity was measured at $450 \mathrm{~nm}$ with an ELISA reader (Spectra Max 190, Molecular Devices, Sunnyvale, CA, USA).

Immunoprecipitation. Protein GHP SpinTrap ${ }^{\mathrm{TM}}$ column (GE Healthcare, Amersham, UK) was washed and equilibrated

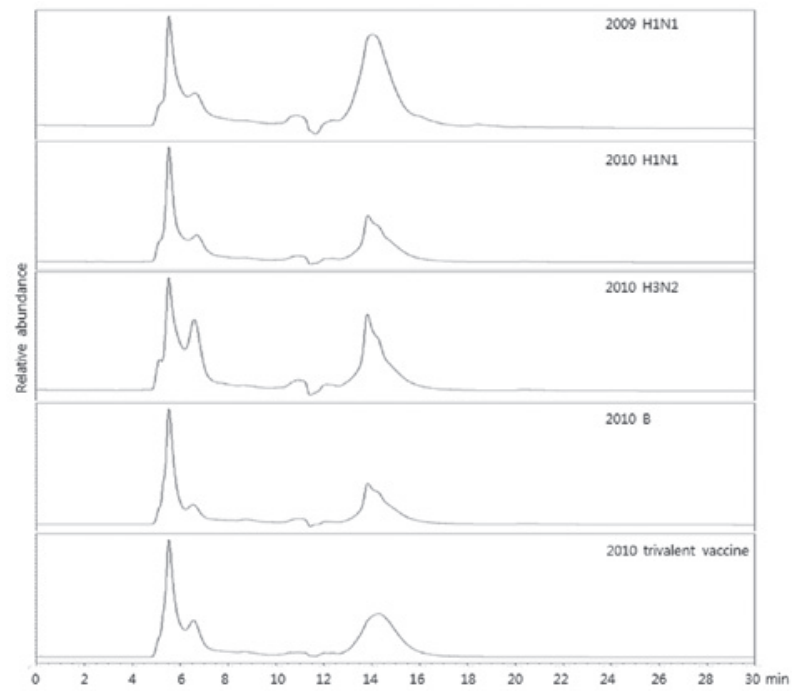

Figure 1. Comparison of SE-HPLC chromatograms of influenza vaccines. SE-HPLC chromatograms of 2009 H1N1, 2010 H1N1, 2010 H3N2, 2010 B monovalent vaccines and 2010 trivalent vaccine were assessed. The SE-HPLC chromatogram pattern was similar among the vaccines manufactured by a single manufacturer under the same process, regardless of virus type. SE-HPLC, size exclusion high performance liquid chromatography.

with binding buffer (1X Tris-buffered saline) following removing the storage solution and reacting with $200 \mu 1$ of anti-H1N1 serum for $30 \mathrm{~min}$. The column was washed with $400 \mu 1$ of binding buffer and $200 \mu 1$ of reference antigen or the influenza vaccine was applied for $1 \mathrm{~h}$. Following centrifugation at $150 \mathrm{x} \mathrm{g}$ for $1 \mathrm{~min}$, the eluted solution was subjected to HPLC to verify the HA peak intensity.

Analysis of the correlation of the HA content between SRID and SE-HPLC. The SRID assessment was conducted to measure the HA content of the influenza vaccines relative to the reference antigens. In addition, the SE-HPLC chromatogram area for the HA content of the reference antigens and influenza vaccines was measured. The SE-HPLC chromatogram area/HA $(1 \mu \mathrm{g})$ was calculated for the reference antigens and influenza vaccines, respectively. The SE-HPLC chromatogram area was calculated and compared with that of the SRID.

\section{Results}

SE-HPLC analysis: HA peak examination by SE-HPLC. To compare the HA content, vaccine samples $(2009 \mathrm{H} 1 \mathrm{~N} 1$, 2010 H1N1, 2010 H3N2 and 2010 B monovalent vaccines and 2010 seasonal trivalent vaccines) were analyzed using SE-HPLC. The result demonstrated that the patterns of chromatogram were similar among the assessed vaccines, meaning that SE-HPLC analysis revealed similar results although a different HA was applied (Fig. 1). To verify the specificity of SE-HPLC, 2009 H1N1 reference antigen was immunoprecipitated with antiserum for H1N1 and peaks for HA were compared prior to and following immunoprecipitation (Fig. 2A). The results revealed that the peaks of SE-HPLC were reduced following immunoprecipitation, suggesting that the peaks from SE-HPLC were correlated with HA antigen. The fractions from each peak were designated 1 to 7 , harvested and subject to an ELISA assay (Fig. 2B). When the fractions were reacted with antiserum for 
Table I. Vaccines and reference reagents.

\begin{tabular}{llll}
\hline Vaccine type & \multicolumn{1}{c}{ Vaccine strain } & Antigen reference code & Antiserum reference code \\
\hline 2009 H1N1 & NYMC-X179A (A/California/7/2009) & NIBSC 09/146 & NIBSC 09/152 \\
2010 H1N1 & NYMC-X181 (A/California/7/2009) & NIBSC 09/294 & NIBSC 09/152 \\
2010 H3N2 & NYMC-X187 (A/Victoria/210/2009) & NIBSC 10/102 & NIBSC 09/270 \\
2010 B & B/Brisbane/60/2008 & NIBSC 08/352 & CBER B-Ab-0913
\end{tabular}

NIBSC, National Institute for Biological Standards and Control; CBER, Center for Biologics Evaluation and Research.

Table II. Content of HA in pandemic influenza vaccine by SE-HPLC using the 2009 H1N1 reference antigen.

\begin{tabular}{|c|c|c|c|c|c|c|}
\hline $\begin{array}{l}\text { Reference } \\
\text { and vaccines }\end{array}$ & $\begin{array}{l}\text { HA content } \\
\text { from SRID } \\
(\mu \mathrm{g} / 0.5 \mathrm{ml})\end{array}$ & $\begin{array}{l}\text { HA peak area } \\
\text { from SE-HPLC }\end{array}$ & $\begin{array}{l}\text { HA peak } \\
\text { area/ } 1 \mu \mathrm{g}^{\mathrm{b}}\end{array}$ & $\begin{array}{c}\text { Normalized HA } \\
\text { peak area }^{c}\end{array}$ & $\begin{array}{l}\text { HA content from } \\
\text { SE-HPLC } \\
(\mu \mathrm{g} / 0.5 \mathrm{ml})^{\mathrm{d}}\end{array}$ & $\begin{array}{l}\text { Similarity } \\
(\%)^{\mathrm{e}}\end{array}$ \\
\hline 2009 H1N1 antigen & 15.00 & 831,671 & 55,445 & & & \\
\hline Lot 1 & 12.05 & 728,850 & 60,485 & 1.09 & 12.29 & 101.95 \\
\hline Lot 2 & 11.52 & 731,220 & 63,474 & 1.14 & 12.33 & 106.99 \\
\hline Lot 3 & 12.72 & 725,524 & 57,038 & 1.03 & 12.23 & 96.14 \\
\hline Lot 4 & 12.99 & 725,642 & 55,862 & 1.01 & 12.23 & 94.16 \\
\hline Lot 5 & 13.07 & 723,723 & 55,373 & 1.00 & 12.20 & 93.34 \\
\hline Lot 6 & 12.80 & 722,592 & 56,453 & 1.02 & 12.18 & 95.16 \\
\hline Lot 7 & 10.87 & 721,421 & 66,368 & 1.20 & 12.16 & 111.87 \\
\hline Lot 8 & 11.10 & 647,786 & 58,359 & 1.05 & 10.92 & 98.37 \\
\hline Lot 9 & 10.85 & 657,194 & 60,571 & 1.09 & 11.08 & 102.10 \\
\hline Lot 10 & 10.86 & 647,892 & 59,659 & 1.08 & 10.92 & 100.56 \\
\hline Average/SD value & & & & $1.07 / 0.06$ & & $99.91 / 5.94$ \\
\hline
\end{tabular}

${ }^{\mathrm{a}}$ Vaccines, 10 different lots of $2009 \mathrm{H} 1 \mathrm{~N} 1$ pandemic vaccines were assessed; ${ }^{b} \mathrm{HA}$ peak area/1 $\mu \mathrm{g}=\mathrm{HA}$ peak area from SE-HPLC/HA content from SRID; 'Peak area of the vaccine was normalized with that of reference antigen; ${ }^{\mathrm{d}} \mathrm{HA}$ Content $(\mu \mathrm{g} / 0.5 \mathrm{ml})=(\mathrm{vaccine}$ SE-HPLC peak area/Reference SE-HPLC peak area) x Reference HA content $(\mu \mathrm{g})$ from SRID/Average of normalized HA peak area; ${ }^{\mathrm{e}}$ Similarity $=(\mathrm{HA}$ content from SE-HPLC)/(HA content from SRID) x 100. HA, hemagglutinin; SE-HPLC, size exclusion high performance liquid chromatography; SRID, single radial immunodiffusion; SD, standard deviation.

H1N1, fractions between 1,2 and 3 peaks exhibited higher absorbance than others. These results suggested that major HA contents may be present in 1,2 and 3 peaks. To confirm the specificity of SE-HPLC, an immunoprecipitation and ELISA assay were also conducted with the $2009 \mathrm{H} 1 \mathrm{~N} 1$ vaccine (Fig. 3A and B). A total of 7 peaks were also designated and assessed and the results were similar to those of Fig. 2.

Correlation of HA content with peak area. To examine the correlation of the HA content according to the SE-HPLC chromatogram area, different concentrations of 2009 H1N1 reference antigen were assessed. The result revealed that the chromatogram area was enhanced proportionately following the increased HA concentration from the 2009 H1N1 reference antigen (Fig. 4) and $2009 \mathrm{H} 1 \mathrm{N1}$ vaccine (Fig. 5; R²>0.99). The present results revealed that the quantitative read-out from the SE-HPLC is matched with the content of HA.

Correlation of SRID with SE-HPLC analyses in the measurement of $H A$ content. To examine the accuracy and similarity of
SE-HPLC compared with SRID, 2009 H1N1 (Table II) and 2010 H1N1 (Table III) vaccines were assessed. Initially, 10 repeats of the $2009 \mathrm{H} 1 \mathrm{~N} 1$ vaccine, together with $2009 \mathrm{H} 1 \mathrm{~N} 1$ reference antigen were examined and the content of HA, analyzed by SRID and SE-HPLC was evaluated. To compare HA content from SRID or SE-HPLC, the peak area from SE-HPLC was applied to the following equation: HA content $(\mu \mathrm{g} / 0.5 \mathrm{ml})=($ vaccine SE-HPLC peak area/reference SE-HPLC peak area) x reference HA content $(\mu \mathrm{g})$ from SRID/average of normalized HA peak area. The HA content of the $2009 \mathrm{H} 1 \mathrm{~N} 1$ vaccines was measured by SRID using the 2009 H1N1 reference antigen. In addition, the HA peak area was measured by SE-HPLC using the same reference antigen and then converted into the HA content using the above equation. In the present results, the similarity of HA content analyzed by SRID and SE-HPLC was $99.91 \%$ (SD, 5.94), meaning that evaluation of HA content by SE-HPLC is as reliable as SRID in $99.91 \%$ of cases. The HA content of the $2010 \mathrm{H} 1 \mathrm{~N} 1,2010 \mathrm{H} 3 \mathrm{~N} 2$ and $2010 \mathrm{~B}$ monovalent vaccines was also measured by SE-HPLC and compared with those of SRID using the 2009 H1N1 reference antigen. The result was 
Table III. Content of HA in monovalent influenza vaccines by SE-HPLC using 2009 H1N1 as a reference material.

\begin{tabular}{lcccccc}
\hline $\begin{array}{l}\text { Reference } \\
\text { and vaccines }^{\mathrm{a}}\end{array}$ & $\begin{array}{c}\text { HA content } \\
\text { from SRID } \\
(\mu \mathrm{g} / 0.5 \mathrm{ml})\end{array}$ & $\begin{array}{c}\text { HA peak area } \\
\text { from SE-HPLC }\end{array}$ & $\begin{array}{c}\text { HA peak } \\
\text { area } / 1 \mu \mathrm{g}^{\mathrm{b}}\end{array}$ & $\begin{array}{c}\text { Normalized HA }^{\mathrm{c}} \\
\text { peak area }^{\mathrm{c}}\end{array}$ & $\begin{array}{c}\text { HA content from } \\
\text { SE-HPLC } \\
(\mu \mathrm{g} / 0.5 \mathrm{ml})^{\mathrm{d}}\end{array}$ & $\begin{array}{c}\text { Similarity } \\
(\%)^{\mathrm{e}}\end{array}$ \\
\hline Reference antigen & 15.00 & 650,164 & 43,344 & & & \\
2010 H1N1 & 14.03 & 899,807 & 64,134 & 1.48 & 14.72 & 104.94 \\
2010 H3N2 & 12.82 & 724,381 & 56,504 & 1.30 & 11.85 & 92.45 \\
2010 B & 14.56 & 917,087 & 62,987 & 1.45 & 15.01 & 103.06 \\
Average (SD) & & & & $1.41(0.09)$ & & $100.00(6.73)$ \\
\hline
\end{tabular}

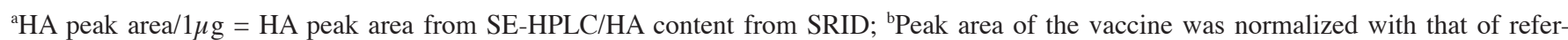
ence antigen; ${ }^{c} \mathrm{HA}$ Content $(\mu \mathrm{g} / 0.5 \mathrm{ml})=($ vaccine SE-HPLC peak area/reference SE-HPLC peak area) $\mathrm{x}$ reference HA content $(\mu \mathrm{g})$ from SRID/average of normalized HA peak area; ${ }^{\mathrm{d}}$ Similarity = (HA content from SE-HPLC)/(HA content from SRID) x 100. HA, hemagglutinin; SE-HPLC, size exclusion high performance liquid chromatography; SRID, single radial immunodiffusion; SD, standard deviation.

A

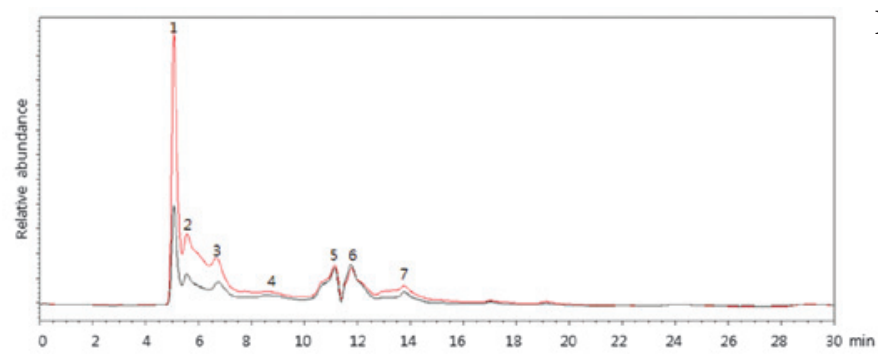

B

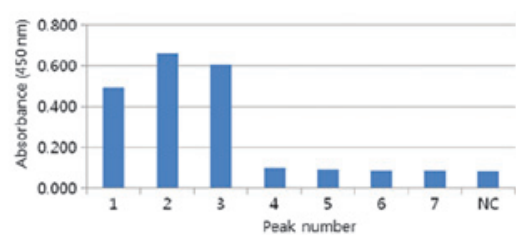

Figure 2. SE-HPLC chromatogram pattern of 2009 H1N1 reference antigen following immunoprecipitation. To examine the specificity of the SE-HPLC peaks for the HA, the $2009 \mathrm{H} 1 \mathrm{~N} 1$ reference antigen was immunoprecipitated with anti-H1N1 serum. (A) SE-HPLC peaks represented before (red line) and after (blue line) immunoprecipitation. (B) The elutes of the SE-HPLC peaks were provided to the ELISA assay for the reaction with anti-H1N1 serum. NC, negative control; SE-HPLC, size exclusion high performance liquid chromatography.

A

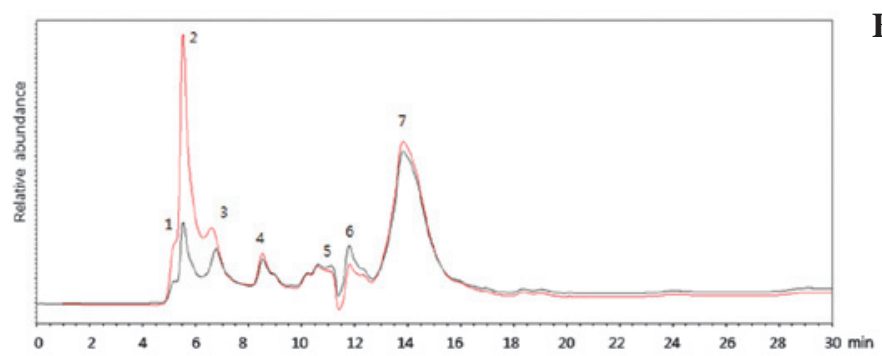

B

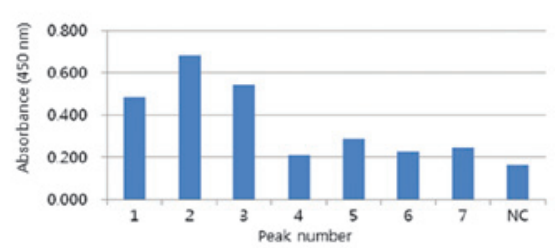

Figure 3. SE-HPLC chromatogram pattern of 2009 H1N1 vaccine following immunoprecipitation. To examine specificity of the SE-HPLC peaks for the HA, the 2009 H1N1 vaccine was immunoprecipitated with anti-H1N1 serum. (A) SE-HPLC peaks represented before (red line) and after (blue line) immunoprecipitation. (B) The elutes of the SE-HPLC peaks were provided to the ELISA assay for the reaction with anti-H1N1 serum. NC, negative control; SE-HPLC, size exclusion high performance liquid chromatography.

similar to that of the $2009 \mathrm{H} 1 \mathrm{~N} 1$ vaccines with $100 \%$ (SD, 6.73) similarity.

\section{Discussion}

The prevention of influenza through vaccination is the most effective method to control an influenza pandemic (14). This has been confirmed once again during the 2009/2010 H1N1 influenza pandemic. In addition, to maximize the preventive effect, vaccination should be performed as soon as possible following the onset of the influenza virus outbreak. For rapid vaccination, the shortening of the time required for vaccine development, including isolation of the pandemic virus, manufacturing of the vaccine virus with high yield, measurement of antigen content and clinical assessment is required $(6,13)$.

At present, the internationally authorized measurement of the antigen content of an influenza vaccine is using SRID. This method measures HA content by comparing the areas of the reference antigen with known HA content and the vaccine. Therefore, for the measurement of the HA content, the reference antigen and antibody are essentially required. It generally takes 2-3 months to prepare the reference antigen and antibody, which is the most significant obstacle in vaccine development. 

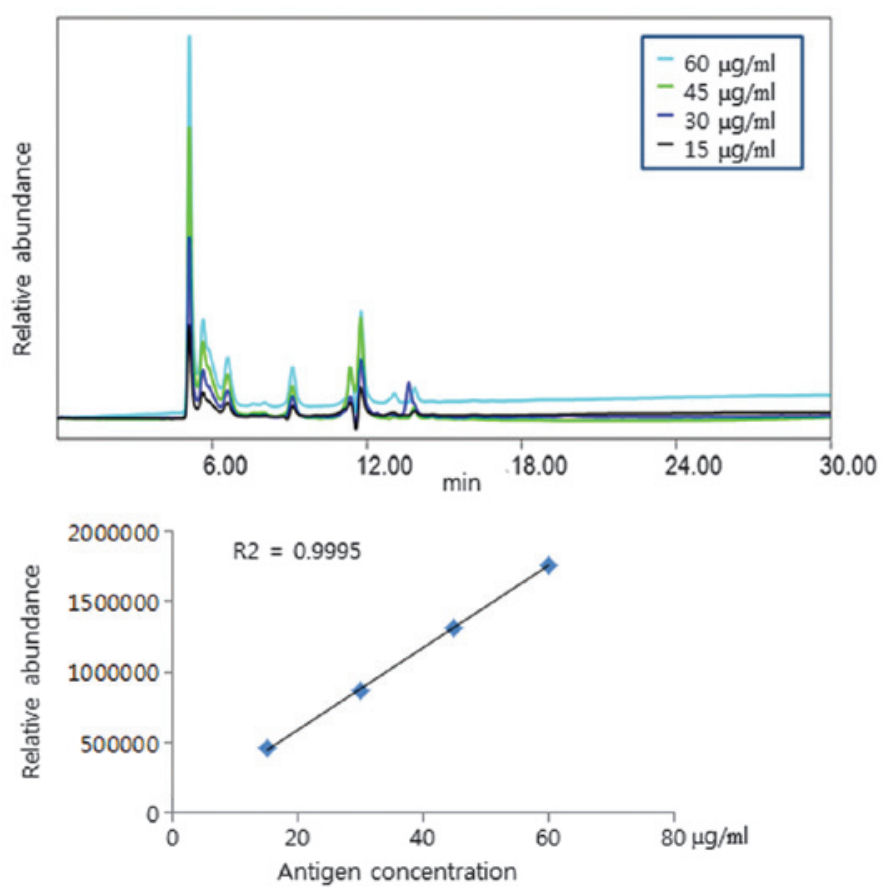

Figure 4. Dose-dependent calibration curve of the $2009 \mathrm{H} 1 \mathrm{~N} 1$ reference antigen. The different concentrations (15, 30, $45 \mathrm{and} 60 \mu \mathrm{g} / \mathrm{ml})$ of the $2009 \mathrm{H} 1 \mathrm{~N} 1$ reference antigen were provided and the SE-HPLC chromatogram area was evaluated. When the HA content of the 2009 H1N1 reference antigen was analyzed by SE-HPLC, the chromatogram area was increased in proportion to the concentration, which revealed a significant correlation $\left(\mathrm{R}^{2}=0.99\right)$. SE-HPLC, size exclusion high performance liquid chromatography.
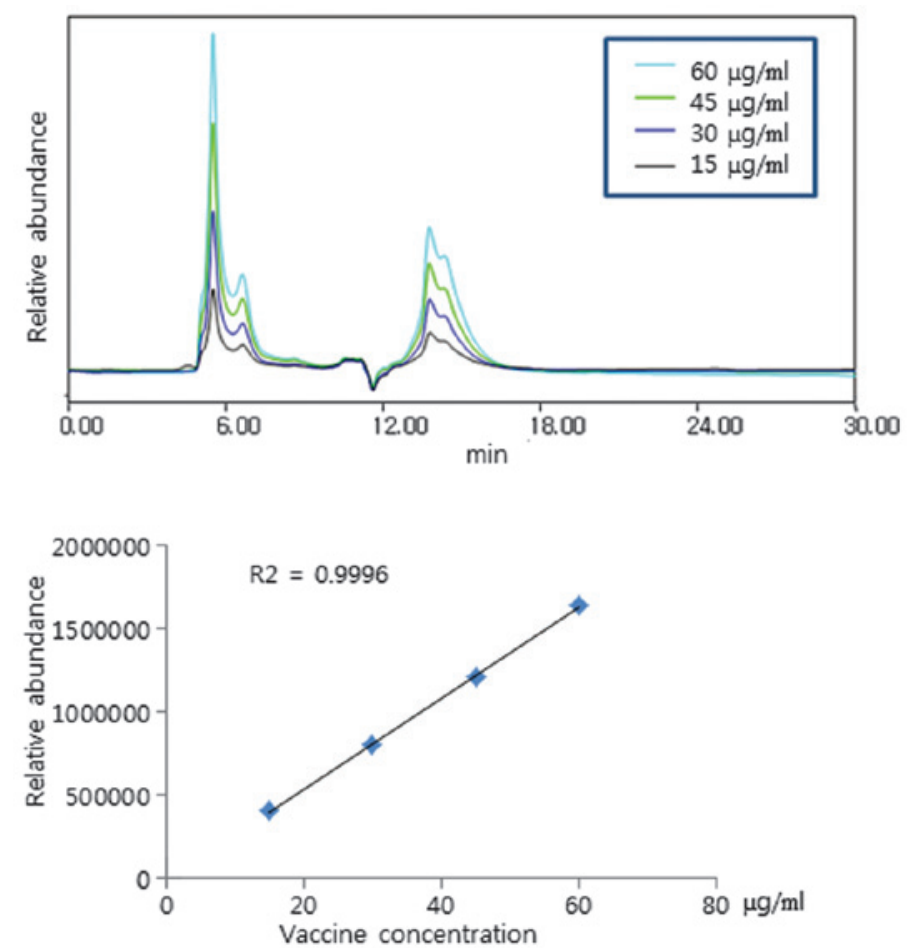

Figure 5. Dose-dependent calibration curve of the $2009 \mathrm{H} 1 \mathrm{~N} 1$ vaccine. The different concentrations $(15,30,45$ and $60 \mu \mathrm{g} / \mathrm{ml})$ of the $2009 \mathrm{H} 1 \mathrm{~N} 1 \mathrm{vaccine}$ were provided and the SE-HPLC chromatogram area was evaluated. When the HA content of the 2009 H1N1 vaccine was analyzed by SE-HPLC, the chromatogram area was increased in proportion to the concentration, which revealed a significant correlation $\left(\mathrm{R}^{2}=0.99\right)$. SE-HPLC, size exclusion high performance liquid chromatography.

The present study was conducted to develop a simple method that measures the HA content without the reference antigen and antibody. The present study revealed a relevant similarity in data produced using SE-HPLC to that of SRID, meaning SE-HPLC may be applied to any type of influenza virus in the situation of an influenza pandemic. The results of SE-HPLC analysis on H1, H3 and B type influenza vaccines revealed similar chromatogram patterns. Furthermore, the 
retention time designating HA separation revealed consistent results with the chromatogram. The SE-HPLC chromatogram area was increased in proportion to HA concentration, which demonstrated a significant correlation between the HA concentration and peak area. Although the HA content, as examined using SE-HPLC did not accurately represent HA antigenicity compared with that of SRID, it exhibited a high similarity to that of SRID (99.91-100.00\%) with regards to HA concentration.

During the SE-HPLC process, protease treatment for the specimens of the reference antigen and the vaccine was not applied, which differs from the SRID method. As the protease step was skipped, the results may not be as accurate as SRID. However, an experimental protocol, which excludes protease treatment saves time in the analysis of the HA content. Therefore, SE-HPLC is not a method that replaces the SRID assay, but a supplementary assessment to overcome delayed vaccine development, a disadvantage of the SRID assay. If a clinical dose of HA content is prepared using SE-HPLC prior to the development of the reference antigen and antibody for a pandemic influenza virus, a shortening of the vaccination development time against the pandemic influenza virus may be achieved. A further study on the influenza pandemic virus type $\mathrm{H} 5$ is required for more accurate measurement.

\section{Acknowledgements}

The authors would like to thank Green Cross for providing influenza vaccines and NIBSC for providing reference materials. The present study was supported by a grant from the Korean Food and Drug Administration (no. 10171KFDA307).

\section{References}

1. Schild GC, Wood JM and Newman RW: A single-radial-immunodiffusion technique for the assay of influenza haemagglutinin antigen. Proposals for an assay method for the haemagglutinin content of influenza vaccines. Bull World Health Organ 52: 223-231, 1975.
2. Wood JM, Schild GC, Newman RW and Seagroatt V: An improved single-radial-immunodiffusion technique for the assay of influenza haemagglutinin antigen: application for potency determinations of inactivated whole virus and subunit vaccines. J Biol Stand 5: 237-247, 1977.

3. Wood JM, Mumford J, Schild GC, Webster RG and Nicholson KG: Single-radial-immunodiffusion potency tests of inactivated influenza vaccines for use in man and animals. Dev Biol Stand 64: 169-177, 1986.

4. Williams MS: Single-radial-immunodiffusion as an in vitro potency assay for human inactivated viral vaccines. Vet Microbiol 37: 253-262, 1993.

5. Hu J, Liu DC and Wang TR: Clinical application of the single radial immunodiffusion (SRID) technic to detect antitubercle bacillus antibody. Zhonghua Jie He He Hu Xi Za Zhi 16: 270-271, 318-279, 1993 (In Chinese)

6. Gerdil C: The annual production cycle for influenza vaccine. Vaccine 21: 1776-1779, 2003.

7. Kapteyn JC, Saidi MD, Dijkstra R, et al: Haemagglutinin quantification and identification of influenza A\&B strains propagated in PER.C6 cells: a novel RP-HPLC method. Vaccine 24: 3137-3144, 2006.

8. Kapteyn JC, Porre AM, de Rond EJ, et al: HPLC-based quantification of haemagglutinin in the production of egg- and MDCK cell-derived influenza virus seasonal and pandemic vaccines. Vaccine 27: 1468-1477, 2009.

9. Garcia-Canas V, Lorbetskie B, Bertrand D and Cyr TD, Girard M: Selective and quantitative detection of influenza virus proteins in commercial vaccines using two-dimensional high-performance liquid chromatography and fluorescence detection. Anal Chem 79: 3164-3172, 2007.

10. Williams TL, Luna L, Guo Z, et al: Quantification of influenza virus hemagglutinins in complex mixtures using isotope dilution tandem mass spectrometry. Vaccine 26: 2510-2520, 2008.

11. Peyre M, Fusheng G, Desvaux S and Roger F: Avian influenza vaccines: a practical review in relation to their application in the field with a focus on the Asian experience. Epidemiol Infect 137: $1-21,2009$.

12. Li C, Shao M, Cui X, et al: Application of deglycosylation and electrophoresis to the quantification of influenza viral hemagglutinins facilitating the production of 2009 pandemic influenza (H1N1) vaccines at multiple manufacturing sites in China. Biologicals 38: 284-289, 2010.

13. Wood JM and Levandowski RA: The influenza vaccine licensing process. Vaccine 21: 1786-1788, 2003.

14. Ferguson NM, Cummings DA, Fraser C, Cajka JC, Cooley PC and Burke DS: Strategies for mitigating an influenza pandemic. Nature 442: 448-452, 2006. 